Biol, 57,623-625.) before. Adding dioxane to the previous buffer system, the author has also obtained perfect crystals of neuraminidase, with space group $P 42,2$.

Some divalent metal ions, particularly metal ions of the transition series have been found that stimulate crystal growth. In order to be free of serious twinning problems in crystal growth of neuraminidase type $B$, we added $\mathrm{CaCl}_{2}$ to the previous buffer system, obtained very ideal crystals and collected a dataset at 2.3A resolution [6](Lin, YuJuan, et al., (1990), J. Mol. Biol. 214, 639-640.).

Decreasing the protein concentration is of great advantage to eliminate crystal twinning. For example, single crystals of Trichosanthin-with $C 2$ space group are often found on the edge of the drop or on end with low protein concentration.

As before, we might draw the conclusion that it is unnecessary to give up the previous buffer system to overcome the crystal twinning if only trying the ways described above.

\section{PS-15.01.09 IMPROVEMENT OF EGF RECEPTOR CRYSTALLIZATION BY EXPOSURE TO MICROGRAVITY AND BY ISOFORM SEPARATION}

E. Wenisch ${ }^{2}$, N. Günther ${ }^{1}$, L.J. DeLucas ${ }^{3}$, C.E. Bugg ${ }^{3}$, P.G. Righetti ${ }^{2}$, Ch. Betzel ${ }^{4} \& W$. Weber ${ }^{4 *}$

1 Universitătskrankenhaus Eppendort, Hamburg, Germany $\cdot{ }^{2}$ University of Milano, Italy . ${ }^{3}$ Center for Macromolecular Crystallography, UAB, Birmingham, Alabama, USA - ${ }^{4}$ EMBL-Outstation, DESY, Hamburg, Germany

The EGF receptor plays an important role in normal and pathological growth control. A secreted form of the receptor (sEGFR) representing its $100 \mathrm{kd}$ external domain (Weber, Gill, Spieß, Science, 1984, 224, 294-7) had been crystallized in the presence of the ligand, and a 1:1 molar complex of receptor and ligand could be recovered in the crystals. Diffraction, however, had been only about $10 \AA$, probably due to the high extent $(30 \%)$ of complex receptor glycosylation and to the limited volume of the crystals obtained so far (Günther, Betzel, Weber, J. Biol. Chem., 1990, 265, 22082-5).

A significant improvement of crystal quality was achieved in a crystallization experiment under microgravity conditions on US space shuttle mission STS47. In 8 out of 9 set-ups prepared under different conditions crystal growth was observed after 8 days in space. One of the space-grown crystals $0.6 \times 0.43 \times$ $0.2 \mathrm{~mm}^{3}$ ) showed higher diffraction quality than all earth-grown crystals before, and it allowed for the first time partial data collection: diffraction extended for the first 5 exposures to $6 \AA$ resolution, followed by a rapid decrease of diffraction patterns occurring during the following exposures. 2585 reflections were merged to R.symm $=9.3 \%$.

A further improvement of crystal quality was realized in the laboratory by a novel technique included as an additional purification step of the protein: Whereas receptor preparations used for crystallization so far, consisted of more than 10 isoforms resolved by isoelectric focusing, selected single pl-species were prepared by exploiting the unique resolving power of a multicompartment electrolyzer with isoelectric membranes (Wenisch, Righetti, Weber, Electrophoresis, 1992, 13,668-73). From one of these isoforms a crystal could be grown to a size of $1.3 \times 0.5 \times$ $0.3 \mathrm{~mm}^{3}$. It allowed data collection up to $6 \AA$ for the first 10 images; then diffraction patterns decreased to $10 \AA$ during the following 10 exposures because of increasing radiation damage. 2942 reflections were merged to R.symm $=5.4 \%$. The reduced data set showed a completeness of $26 \%$.

The space group of both, the space-grown 'wild-type'-receptor as well as the earth-grown single-pl isoform, was assigned to be orthorhombic $\mathrm{P} 2,22$ or $\mathrm{P} 2{ }_{1} 2_{1} 2$ with pseudo-tetragonal unit cell parameters of $a=116.3 \AA, b=119.5 \AA$, and $c=204.5 \AA$. These values yield a unit cell volume of $2.8 \times 106 A^{3}$ and $a$ packing parameter $V_{M}$ of $3.58 \AA^{3} /$ Dalton assuming 2 molecules each of receptor and ligand in the asymmetric unit. The fractional volume occupied by solvent was calculated to be $65 \%$.

Since either approach, microgravity conditions and isoprotein selection, has improved crystal growth, further benefit may be expected from a combination of both: crystallization of selected EGF receptor isoforms in space.

\subsection{2 - Crytal Growth of Inorganic Compounds}

PS- 5 5.02.01 RAPID GROWTH OF TGS AND ATGSP CRYSTALS By Wang Qingwu*, Fang Changshui, zhuo Hongsheng and Wang Min, Institute of Crystal Materials, Shandong University, Jinan 250100, P.R.C.

At present, TGS (triglycine sulphate) series crystals are excellent pyroelectric materials being widely used to make infrared detectors and vidicons. We have engaged in the study of the rapid growth of these crystals. First, we studied the solution status of TGS and ATGSP (L-alanine and phosphoric doped TGS) crystals and selected the optimum solution conditions for rapid growth. By adding a rapid rotating impeller to enhance the convection of solution in the crystal growth process, we have realized the rapid growth of these crystals. The growth rate of the (010) face, whose cross section is $40 \mathrm{~mm} 40 \mathrm{~mm}$, reached $5 \mathrm{~mm} /$ day by using single face growth technique. The growth rate has been raised 3-5 times of the normal rate.

Second, we studied the perfection of the asgrown crystals by $x$-ray topography, and found that there are more defects in the rapidgrowth crystals than those grown by normal speed.

Finally, the properties of TGS and ATGSP crystals grown by using high speed have been measured. The results show that pyroelectic coefficient and dielectric constant of the rapid-growth crystals are icreased slightly, but their figures of merit are almost the same as those of the crystals grown by normal speed. Especially in the case of the ATGSP crystal, when its growth rate is $5 \mathrm{~mm} / \mathrm{day}$, its biag field reached $10 \mathrm{Kv} / \mathrm{cm}$, and its dielectric loss at roon temperature is 2-3 times smaller than that of the crystals grown by rormal speed. The peak of dielectric loss near the curie point disappears. These changes are due to the high bias field in crystals. The high bias field and low dielectric loss are very important for making infrared detectors and vidicons. 